\title{
Empathy Study in Rodent Model of Autism Spectrum Disorders
}

\author{
Tamanna Jahan Mony ${ }^{1}$, Minha Hong ${ }^{2}$, and Hee Jae Lee ${ }^{1 凶}$ \\ ${ }^{1}$ Department of Pharmacology, School of Medicine, Kangwon National University, Chuncheon, Republic of Korea \\ ${ }^{2}$ Department of Psychiatry, School of Medicine, Seonam University, Goyang, Republic of Korea
}

\begin{abstract}
There is a highly cognitive and social context to empathy behavior in human. In various social contexts, rodents also display remarkable affective sensitivity and exhibit primitive forms of empathy similar to human. Therefore, we aimed to elaborate the concept of empathy about various components of empathetic behavior in rodents with the similar contexts of a human. In this review, we highlighted the behavioral paradigm that already examined different aspects of rodent empathetic behavior in response to conspecific distress. Additionally, we summarized homologous brain parts of human and rodents to express the empathetic behavior. Integrating the findings with corresponding experiments in the human will provide a novel insight into therapeutic intervention or expanded experimental approaches for neuropsychiatric disorders like autism spectrum disorders associated with empathetic behavior.
\end{abstract}

Psychiatry Investig 2018;15(2):104-110

Key Words Empathy, Autism spectrum disorders, Rodent.

\section{INTRODUCTION}

It is inevitable that all mammalian brains capable of generating felt and emotional experiences. This contention has been speculated for years but only recently has revealed as foundational. Empathy has been considered as a high level of affective and cognitive process, expressed exclusively by the human for social living. It is a crucial part of a human emotional experience and social interaction. ${ }^{1}$ The ability of one person to perceive what another person is feeling and thinking is considered to be inimitable to human alone and may not be inherent to the lower mammalian range like rodents.

However, recent reports support the possibility that rodents also display elements of empathy, i.e., showing to emotion to others..$^{2-4}$ Rodents illustrate emotional reactions to the pain of conspecific and different social factors can alter their pain sensitivity. ${ }^{5}$ Most of the studies reported analgesia and did not able to measure the effects in the actual moment when another's pain was exactly being perceived. ${ }^{6}$ These phenomena may

Received: February 1, 2017 Revised: May 14, 2017

Accepted: June 20, 2017 Available online: December 14, 2017

$\triangle$ Correspondence: Hee Jae Lee, $\mathrm{PhD}$

Department of Pharmacology, School of Medicine, Kangwon National University, 1 Kangwon Univ-gil, Chuncheon 24289, Republic of Korea Tel: +82-33-250-8850, E-mail: heejaelee@kangwon.ac.kr

(a) This is an Open Access article distributed under the terms of the Creative Commons Attribution Non-Commercial License (http://creativecommons.org/licenses/bync/4.0) which permits unrestricted non-commercial use, distribution, and reproduction in any medium, provided the original work is properly cited. represent an example of the coaction of social facilitation.

Empathy is currently a topic of study in human ${ }^{6,7}$ and extensive human literature documented that the effects on pain feeling may be mediated precognitively. ${ }^{1}$ The complex cognitive abilities are evaluated in rodents using cognitive tests. Cognition depends on medial frontal cortex connections, such as the intra-dimensional and extra-dimensional attentional set of alteration task. ${ }^{8,9}$ Executive functions are demanding simultaneously the amalgamation of large amounts of complex information (social and non-social) that assumed to localize in the prefrontal cortex. ${ }^{10}$ Prefrontal cortex region of brain part is not well developed in rodents or others as like as human brain. ${ }^{11}$ There are clear limitations of the mechanistic information that can be assembled from animal studies. Like as eye gaze is difficult to track in rodent, as the pupil is hard to distinguish. ${ }^{12}$ Even some elements of joint attention and attentional focus in rodent model need to constant consideration tasks such as the five-choice serial reaction time test with auditory-visual or olfactory distracters. ${ }^{12,13}$ Besides that other features such as in autism spectrum disorder (ASD) studies, like with the 4:1 male: female ratio as well as relapse of social communication after one year of age, certain features are yet difficult to recognize in a rodent model. ${ }^{10}$

Intricacies of language are also unlikely to be instinctive to a rodent although the complexity of rodent ultrasonic vocalization patterns may contain important communicative information. ${ }^{5}$ Quantitative evaluation of the reward value of social 
communication is not yet available for rodents or autistic individuals. Although there are some established models to evaluate or work with the autistic symptom, newer and better models are required.

Designing a behavioral model specifically relevant to human mental disorders presents a difficult challenge as there is a discontinuity between human social interactions and those displayed by lower mammals including rodents. Also, the autistic symptoms are unique and inherently variable in humans. ${ }^{10}$ Diagnosis of ASD is mostly based on behavioral criteria with no consistent biological markers available; other relevant etiological factors have also not been identified. As such, effective animal models should incorporate strong analogies to the endophenotypes of the human syndrome. These models should also have the same biological dysfunction that causes the disease (gene mutations or anatomical abnormality) along with having the same corresponding responses in the models to treatments that inhibit or reverse symptoms in humans. ${ }^{11,14}$

Even with smaller prefrontal areas, empathy is evolutionarily conserved in lower mammals as well since rodents are also perfectly capable of showing empathy-related behaviors such as emotional contagion and prosociality. The understanding of self and others as being separate individuals is essential for producing noble behaviors (e.g., prosocial and helping behaviors), and the existence of such social abilities has been considered an extraordinary phenomenon in non-primate animals. The availability of an animal model of empathy will allow the application of additional experimental techniques for heterogeneous neurodevelopmental disorders like ASD.

Though there is strong evidence that rodents display empathy-related behaviors, the detailed understanding of the neural mechanisms involved remains unclear, and more studies are required for a better understanding of rodent empathy, having the potential to provide a distinct outline for understanding these highly important, complex and interesting behaviors. We aimed that this review on the subject will support the application of this new translational model to ASD research.

\section{WHAT IS EMPATHY?}

Empathy is a product of an integrated set of brain processes. ${ }^{11}$ Empathy includes a level of cognitive ability that varies according to social, temporal and environmental contexts. In these contexts, emotions are a primary substrate for all empathetic manifestations. The word "empathy" has been defined as the perception of an emotional signal in the other individual, as it directly stimulates a similar reaction in the observer, lacking any intervening, labeling, associative or cognitive perspective processes such as talking or physical interactions. ${ }^{15}$
A very practical definition of empathy proposed by Vignemont and Singer within an experimental context. In the definition, they explained that empathy happens when an individual (A) experiences an emotional stage that is isomorphic to the emotional stage of another and is incited by discerning or evoking the particular expression of the emotive state by an individual (B). ${ }^{16}$ Therefore the affective state of the individual (B) become the source of emotional change in individual (A). ${ }^{16}$

There has been more recent research on relating how an individual comes to recognize another's pain and is able to distinguish the condition of the other from its own, along with feeling the emotional state of the other even though they are not sharing the same state. "Primal empathy" refer to states such as emotional contagion and expressive resonance in which there is an association of depending on affective states between individuals. ${ }^{11,17}$ Primal empathy is a shared neurobehavioral and neuro-affective process, and it is not a unique emotional state.

In humans and including other mammals, primal empathy may relate to higher cognitive functions that allow feelings such as concern or sympathy to develop. Also, "Theory of the Mind" can be defined as the ability to recognize that the perspective of another individual is different from one's own.$^{18}$ It is thought that this type of cognitive empathy allows for an individual to recognize the emotional form of another without necessarily sharing the same emotional situation. ${ }^{19}$

It is important to clearly define the term "empathy" in applying it to cross-species research. "Empathy" also includes using the terms "understand," "recognize," and "image" as those words usually are reserved to describe higher cognitive functions that are difficult to study in animals. Clear cross-species approaches in studying the neural origins of primal empathy will clarify the understanding of the higher forms of empathy as in humans.

\section{BRAIN PARTS INVOLVED IN REGULATING EMPATHETIC BEHAVIOR}

Empathy depends upon share of feelings and emotion. ${ }^{20}$ Mammalian brains have at least seven systems that regulate the unconditioned autonomic and behavioral displays of emotions ${ }^{17,21-25}$ As Lipps, first speculated that so far 'empathy' depends upon share feelings status. ${ }^{19}$ Affective neuroscience studies in cross-species provide a perspective on understanding empathy in higher mammals by concurrently delimiting the behavioral inbred and subjective-experiential features of essential emotional arousal, namely the primal brain reward and punishment systems. ${ }^{17,21,26,27}$

The anatomical circuits for this subcortical emotion gener- 
ating systems originate in primeval medial regions of the upper brainstem that are preserved across mammalian species. ${ }^{17,28-31}$ Stimulation of this system is subjectively experienced, as evidenced by "rewarding and punishing" effects, thereby facilitating the "learning and memory" as secondary processes, as well as "thinking," "ruminating" and other higher mental abilities as tertiary processes. ${ }^{17,28}$

The prefrontal cortex (PFC) is implicated in a wide range of behaviors, as it is connected to different cortical and subcortical parts of the brain. ${ }^{32}$ As they are involved in processing working memory, decision making, goal-directed behavior and social behavior Nucleus accumbens, amygdala, ventral tegmental area, and hypothalamus together built the social brain region. ${ }^{32-34}$ The brain parts of human involve in expressing empathetic behavior are presented in Table 1.

Different studies ${ }^{32,35-37}$ have indicated that different regions of human PFC are homologous to the rodent PFC. There are some dissimilarities between human and rodent structures as well: rodents do not have a granular PFC, as the granular regions of human PFC, including dorsolateral-PFC. ${ }^{32}$ However, the overall similarities of human and rodent $\mathrm{PFC}$ regions might allow for a commonality in social cognition across different mammalian species. The homologous parts of the human and rodent brain are presented in Table 2.
An interesting line of translational research aims to study empathy behavior in the rodent model, based on two behavioral paradigms. One of the two behavioral models is the ability of rodents in modifying their behavior by recognizing conspecifics, and the other is the prosocial behavior paradigm. For the assays, rats are placed in a condition where they have the chance to free a confined conspecifics in the presence of a rewarded food source. ${ }^{38}$ This result provides a strong evolutionary advantage to social species where observational learning and emotional contagion are the basis of empathy as in rodents. In other studies, these behavioral paradigms have provided evidence that this type of learning involves brain regions within the medial PFC (mPFC) as well as anterior cingulate cortex (ACC) in both human and rodents. ${ }^{39,40}$

Mice acquire a conditioned contextual fear by observing conspecifics, and this behavior was found to be dependent on the right ACC. ${ }^{41}$ Separately, when antipsychotic haloperidol or serotonin was injected into the mice ACC, it decreased the expression of conditioned fear in an observational fear learning task..$^{37}$ It was thought that as serotonin microinjection reduced gamma band activity, it might modulate ACC activity and disrupt social behavior. It was also found that mice learned more quickly to lever press for rewarded food when they observed a well-trained demonstrator but this advantage was

Table 1. Different brain regions of human that associated with empathetic behavior

\begin{tabular}{|c|c|c|c|}
\hline & Brain parts & Associated function & References \\
\hline$\overline{\mathrm{A}}$ & $\begin{array}{l}\text { Medial region of prefrontal cortex } \\
\text { (PFC) }\end{array}$ & $\begin{array}{l}\text { These parts is mainly related to social behaviors } \\
\text { associated with the perception of others mainly } \\
\text { with perceptions of self and similar others }\end{array}$ & $\begin{array}{l}\text { Amodio and Frith 2006; Mitchell 2009; } \\
\text { Mitchell et al. 2006; Johnson et al. 2002 }\end{array}$ \\
\hline $\mathrm{B}$ & $\begin{array}{l}\text { Dorso-lateral region of PFC and } \\
\text { ventral PFC }\end{array}$ & $\begin{array}{l}\text { Usually remain active for social tasks, involve } \\
\text { in perception of others as well as cooperation }\end{array}$ & Amodio and Frith 2006; Mitchell et al. $2006^{61,63}$ \\
\hline $\mathrm{C}$ & Dorso-medial PFC & $\begin{array}{l}\text { This region is involved in the perception } \\
\text { of the situation of others and is more directly } \\
\text { connected with recognizing the state of self and } \\
\text { a similar read in others }\end{array}$ & $\begin{array}{l}\text { Bicks et al. 2015; Johnson et al. 2002, } \\
\text { Mitchell 2009; Wise 2008 }\end{array}$ \\
\hline $\mathrm{D}$ & Medial ventral region of PFC & $\begin{array}{l}\text { Involve in special reward and punishment and } \\
\text { motivation and value }\end{array}$ & deQuervain et al. 2004; Kohls et al. $2012^{40,43}$ \\
\hline $\mathrm{E}$ & $\begin{array}{l}\text { Anterior insula (AI), } \\
\text { Inferior frontal gyrus (IFG) } \\
\text { Midcingulate cortex (ACC/MCC) }\end{array}$ & $\begin{array}{l}\text { Express empathy for pain Plays a major role } \\
\text { in representing and integrating internal and } \\
\text { emotional feeling states }\end{array}$ & $\begin{array}{l}\text { Bernhardt and Singer 2012; Lamm et al. 2011; } \\
\text { Craig 2004 }\end{array}$ \\
\hline
\end{tabular}

Table 2. Summary of homologous brain parts of human and rodents to express empathetic behavior

\begin{tabular}{|c|c|c|}
\hline Brain region of human & Homologous region of rodents & References \\
\hline $\begin{array}{l}\text { Prefrontal Cortex (PFC) medial } \\
\text { PFC (both vmPFC \& dmPFC) }\end{array}$ & $\begin{array}{l}\text { Both vmPFC \& dmPFC is also considered to } \\
\text { be homologus to the rodent infralimbic cortex }\end{array}$ & $\begin{array}{l}\text { Bicks et al. 2015; Jeon et al. 2010; } \\
\text { Jurrado-Parras et al. 2012; Kim et al. } 2014^{32,35-37}\end{array}$ \\
\hline Medial orbitofrontal cortex & Rodent medial PFC & $\begin{array}{l}\text { Bicks et al. 2015; Jeon et al. 2010; } \\
\text { Jurrado-Parras et al. 2012; Kim et al. 2014 }\end{array}$ \\
\hline $\begin{array}{l}\text { Dorsal anterior cingulate cortex } \\
\text { (ACC) }\end{array}$ & Rodent ACC & $\begin{array}{l}\text { Jeon et al. 2010; Kim et al. 2014; Kim et al. 2012; } \\
\text { Singer et al. } 2004^{35,37,41,67}\end{array}$ \\
\hline
\end{tabular}


abolished when mPFC was electrically stimulated during the observational learning. ${ }^{36}$

Social hierarchy is another supportive paradigm for ASD studies in rodents. As social hierarchies are common and an important adaptation to living for mammals groups. In higher mammals (for both human and nonhuman primates), a dominance hierarchy involves an understanding of the dominance relationships, reading the intention of others as well as learning the social norms. ${ }^{42}$ In mice, the microcircuitry in the PFC was also linked to the dominance behavior. For example, shifting the efficacy of synaptic transmission in the PFC may cause a bidirectional modulation of the social hierarchy. ${ }^{43,44}$ Wang et $\mathrm{al}^{45}$ also demonstrated the opposing regulation of aggressive and dominance as an activity of the mouse PFC. According to other studies, optogenetically activating the mPFC (prelimbic/infralimbic) in mice decreases aggressive behavior, ${ }^{36}$ and silencing of that region leads to an escalation of aggression. ${ }^{41}$ Knocking out of disk large-associated protein 2 (dl gap2), an important postsynaptic density (PSD) scaffolding protein associated with ASD, increased dominance and aggressive behavior, and it also decreased $\alpha$-amino-3hydroxy-5-methyl-4-isoxazole propionic acid receptor-miniature endplate currents (AMPAR-mEPCSs) and spine density in the mouse orbitofrontal cortex (OFC) ${ }^{41,46}$ Thus, social hierarchy is also modulated by excitatory neurotransmission in PFC, and this could be useful for investigating social cognition alterations in genetic animal models of human ASD.

\section{EMPATHY STUDY IN RODENT MODEL IN DIFFERENT CONTEXT}

Various recent experimental studies demonstrated that rodents were capable of sharing emotional status with others. Here, we will highlight several models that examine different aspects of rodent empathetic behaviors in response to various distress conditions. Along with numerous modern studies relevant to rodent empathy, some foundational studies pointed to the extent to which a rodent could be in concurrence with the affective state of a social partner. There have been two fundamental studies conducted by Church 2 and separately Rice and Gainer 3 that represented as a base for empathy study in rodents. Church demonstrated the behavior of well-trained rats and their performance with a leverpressing task as the rats were concurrently exposed to a conspecific of being shocked. In his study, Church found that a rat could identify whether its emotional experience was temporally coordinated with the same experience in a conspecific. $^{2}$ In the Rice and Gainer ${ }^{3}$ study, they asked whether a rat could exhibit the helping behavior to alleviate the distress of a conspecific. Their work confirmed that rats would keenly work (i.e., help) to reduce the pain of conspecifics, which is another phenomenon that is relevant to empathy.

Social modulation of pain, work conducted by Langford et al. ${ }^{6,47}$ is considered as one of the primary studies that assessed empathy in rodents within the framework of existing neuroscience approaches. Several traditional behavioral assays were incorporated in this study that is applied to evaluate pain in mice. The studies were for understanding social mechanisms in mice where a "partner" was subjected to a painful stimulus. The results of the study demonstrated that mice were truly reactive to the level of pain experienced by other individuals. Langford and co-workers asked for a best conceptualized behavioral finding in which the behavioral change of an individual spontaneously motivated the same state in neighboring conspecifics. ${ }^{48}$ Langford et al. ${ }^{47}$ stated that this behavior in mice was strongly predisposed by the degree of familiarity between the social partners that resembled a human empathy situation. Wöhr and Schwarting in their study assessed the situation as an emotional contagion for rodents responding to the freezing behavior expressed by a nearby demonstrator when tested in a fear-conditioning paradigm. ${ }^{45}$ A series of studies ${ }^{6,49}$ provided strong evidence that emotional contagion could be studied in mice and communication between rodents alter their future capability to learn about emotionally related actions.

More recent studies like that of Bredy and Barad ${ }^{54}$ demonstrated that social interaction with familiar conspecifics who had been previously exposed to the same fear conditioning could modify the acquisition, retention, and extinction of a cued-fear association. The finding of the experiment demonstrated that the experience of previously exposed distress facilitated both the conspecifics to learn the avoidance behavior during the training phase, as well as increased retaining of the fear memory. That was documented by the increased avoidance behavior after 24 hours of distress experience. Extending on these studies, Knapska et al. ${ }^{51}$ conducted a contextual fear-conditioning paradigm experiment. Knapska et al. ${ }^{51}$ found that interacting with a fear-conditioned demonstrator increased retention of memory of a passive behavioral response (freezing) in this paradigm. Although there were certain differences between these two studies, the overall findings demonstrated that the social experience of distress in others could help rodents learn about fearful situations in the future. ${ }^{11}$ They conducted all additional in-depth analysis of social interactions with a distressed conspecific. When rodents were brought together with a newly distressed conspecific, they showed more than ten-fold increase in allogrooming behavior towards the rats compared to that were reunited with a non-distressed conspecific. "Allogrooming" is considered a kind of social activity in rodents, in which individual rodents recur- 
rently lick the head/neck/mouth/ear area of another individual. ${ }^{11}$ It is interesting to speculate that previous studies for primate also suggested that the facilitation behavior after distinguishing the distress of another is an attempt to relief the afflicted individual. ${ }^{15}$ Interestingly, a mutual and heightened stimulation was documented to lead to the induction of transcription factor c-fos in the amygdala of both distressed demonstrator and the observer, and this was shown to increase in the amygdala central nucleus of both groups. This finding suggested that particular sectors of the amygdala are responsiveness to distress stimuli. ${ }^{52}$ It seems that a corresponding set of alterations in the amygdala are involved in numerous emotional processes.

Using a similar approach to the above two studies, Guzman et al. ${ }^{53}$ showed that conditioned stimulus-unconditioned stimulus (CS-UCS) association could regulate the subsequent learning when an individual rat observed the situation of a non-fearful demonstrator. In the behavior model, when the observers were trained in the same condition, the condition of fearful demonstrators did not alter the subsequent learning of the observer. Usually, if mice observed a nonfearful demonstrator, it inhibited their ability to evoke the CS-UCS association memories by themselves. Unlike Bredy and Barad ${ }^{54}$ and Knapska et al..$^{51}$ observer mice in the study of Guzman et al. ${ }^{53}$ were able to derive specific information about the experience of the respective demonstrators.

Ben-Ami Bartal et al..$^{38}$ pioneered in providing the first robust paradigm for the study of pro-social behavior in rats. They demonstrated that a rodent form of empathy was the motivation for this helping behavior. In their study, they found that rats learned to release cage mates trapped in a restrainer, even when they received no explicit reward. Once learned, this behavior was performed consistently and quickly. Surprisingly, the rats liberated a cage mate equally when there was another restrainer containing the same preferred reward item of chocolate chips even when they shared the chocolate with their freed cage mate. In these cases, sex difference was consistent with human studies of gender difference in empathy and social perception; i.e., female rat performed better in this dooropening task. As such, the findings all provided evidence that the rats possessed a shared mechanism in mobilizing pro-social stimulus in response to the distress of others. ${ }^{38}$

With their other subsequent studies, Ben-Ami Bartal et al. ${ }^{38}$ suggested that familiarity not affects the helping behavior. Moreover, the authors found that rats displayed the helping behavior to cage mates even being from a different strain but not to a stranger from the different strain. These studies suggested that strain identity without a social experience not be decisive for prosocial behavior. These findings suggested that experimental approaches using rodent behavioral models could help in elucidating empathy in rodents along with the neurochemical associations. Integrating these results with similar experiments in humans will ultimately provide novel insights into therapeutic interventions for mental disorders associated with empathy.

\section{DISCUSSION}

There is no standard and even no specific diagnosis criteria for ASD. ${ }^{10,55-57}$ These heterogeneous neurodevelopmental disorders presenting a major challenge to designing the predictive validity into translational testing of compounds in the rodent model of ASD. ${ }^{10,52,58-60}$ In this review we screened out the approaches towards generating the reliable experimental approaches for the diagnostic and therapeutic intervention in rodent ASD model. In this review, we tried to provide an accepted definition of "empathy," and justify the feasibility of having an empathy paradigm for ASD research. Demonstrating sympathy and displaying a helping behavior towards a conspecific were found to be core tactics for rodent empathetic behavior. Based on this simple and concise definition of "empathy" in a rodent, this translational model allows for the development of elaborate and powerful experimental techniques in research. In this review, we also focused common underlying mechanisms associated empathetic behavior both in human and rodents. As a part of this analysis, for example, we reviewed several studies that point to the PFC of the brain part as the vital region for modulating empathetic behavior. With this review, we elaborate on modulation of the microcircuitry of the PFC as a key focus for further experimental approaches both at the molecular and neural level. We also reviewed fundamental studies in rodents, providing different contexts for studying empathetic behavior in test animals with applications in ASD research.

\section{FUTURE DIRECTION}

ASD is an unusual neurodevelopmental disorder. Because of its unknown etiology, diagnosis of this disorder is usually based on a set of behavioral criteria. ${ }^{60}$ As the diagnostic criteria for ASD are behavior related, using behavioral assays for a rodent paradigm is pivotal, and it should have a high relevance for each category of the diagnostic symptoms in human. ${ }^{59,60}$ Behavioral neuroscientists are working with an inclusive set of assays related to social interaction, communication, and repetitive behaviors to test the hypotheses on the causes of ASD.$^{10}$ Designing translational models comprised of behavioral assays in test laboratory animals that provide meaning, applicable information is a substantial challenge..$^{58}$ The translational model should have high relevance to the human diag- 
nostic symptoms of ASD. ${ }^{52}$ While designing such models, more facts need to be considered, as the rodent brain is not as well developed as the one for a human, capable of simultaneous integration and functional execution of complex social and nonsocial information.

\section{CONCLUSION}

We expect that the translational research on rodent empathy will allow for bridging the gap between high relevance cross-species social behavior research and understanding ASD; this type of research will also allow for integrative studies in behavioral diagnostic approaches to ASD.

\section{Acknowledgments}

The study was supported by 2015 Research Grant from Kangwon National University.

\section{REFERENCES}

1. Keum S, Shin HS. Rodent models for studying empathy. Neurobiol Learn Mem 2016;135:22-26.

2. Church RM. Emotional reactions of rats to the pain of others. J Comp Physiol Psychol 1959;52:132-134.

3. Rice GE, Gainer P. "Altruism" in the albino rat. J Comp Physiol Psychol 1962;55:123-125.

4. Riess D. Vicarious conditioned acceleration: successful observational learning of an aversive Pavlovian stimulus contingency. J Exp Anal Behav 1972;18:181-186.

5. Scattoni ML, Ricceri L, Crawley JN. Unusual repertoire of vocalizations in adult BTBR T+tf/J mice during three types of social encounters. Genes Brain Behav 2011;10:44-56.

6. Langford DJ, Tuttle AH, Briscoe C, Harvey-Lewis C, Baran I, Gleeson $\mathrm{P}$, et al. Varying perceived social threat modulates pain behavior in male mice. J Pain 2011;12:125-132.

7. Jackson PL, Rainville P, Decety J. To what extent do we share the pain of others? Insight from the neural bases of pain empathy. Pain 2006;125:5-9.

8. Brigman JL, Bussey TJ, Saksida LM, Rothblat LA. Discrimination of multidimensional visual stimuli by mice: intra- and extradimensional shifts. Behav Neurosci 2005;119:839-842.

9. Papaleo F, Crawley JN, Song J, Lipska BK, Pickel J, Weinberger DR, et al. Genetic dissection of the role of catechol-O-methyltransferase in cognition and stress reactivity in mice. J Neurosci 2008;28:8709-8723.

10. Silverman JL, Yang M, Lord C, Crawley JN. Behavioural phenotyping assays for mouse models of autism. Nat Rev Neurosci 2010;11:490-502.

11. Panksepp JB, Lahvis GP. Rodent empathy and affective neuroscience. Neurosci Biobehav Rev 2011;35:1864-1875.

12. Humby T, Wilkinson L, Dawson G. Assaying aspects of attention and impulse control in mice using the 5-choice serial reaction time task. Curr Protoc Neurosci 2005; Chapter 8:Unit 8.5H.

13. Wrenn CC, Turchi JN, Schlosser S, Dreiling JL, Stephenson DA, Crawley JN. Performance of galanin transgenic mice in the 5-choice serial reaction time attentional task. Pharmacol Biochem Behav 2006;83:428440 .

14. Crawley JN. Translational animal models of autism and neurodevelopmental disorders. Dialogues Clin Neurosci 2012;14:293-305.

15. Preston SD, de Waal FB. Empathy: its ultimate and proximate bases. Behav Brain Sci 2002;25:1-20; discussion 20-71.

16. de Vignemont F, Singer T. The empathic brain: how, when and why? Trends Cogn Sci 2006;10:435-441.

17. Panksepp J, Panksepp JB. Toward a cross-species understanding of em- pathy. Trends Neurosci 2013;36:489-496.

18. Farrow TFD, Woodruff PWR. Empathy in Mental Illness. Cambridge: Cambridge University Press, Cambridge University Press; 2007.

19. Lipps T. Einfühlung, innere Nachahmung, und Organepfindungen. Archiv für die gesamte Psychologie 1903;1:185-204.

20. de Waal FB. The antiquity of empathy. Science 2012;336:874-876.

21. Northoff G, Wiebking C, Feinberg T, Panksepp J. The 'resting-state hypothesis' of major depressive disorder - a translational subcortical-cortical framework for a system disorder. Neurosci Biobehav Rev 2011;35: 1929-1945.

22. Decety J, Norman GJ, Berntson GG, Cacioppo JT. A neurobehavioral evolutionary perspective on the mechanisms underlying empathy. Prog Neurobiol 2012;98:38-48.

23. Decety J. The neurodevelopment of empathy in humans. Dev Neurosci 2010;32:257-267.

24. Decety J, Jackson PL. The functional architecture of human empathy. Behav Cogn Neurosci Rev 2004;3:71-100.

25. Decety J, Moriguchi Y. The empathic brain and its dysfunction in psychiatric populations: implications for intervention across different clinical conditions. Biopsychosoc Med 2007;1:22.

26. Bernhardt BC, Singer T. The neural basis of empathy. Annu Rev Neurosci 2012;35:1-23.

27. Keysers C, Gazzola V. Expanding the mirror: vicarious activity for actions, emotions, and sensations. Curr Opin Neurobiol 2009;19:666-671.

28. Panksepp J. Affective Neuroscience: The Foundation of Human and Animal Emotions. New York: Oxford University Press; 1998.

29. Panksepp J. Affective consciousness: core emotional feelings in animals and humans. Conscious Cogn 2005;14:30-80.

30. Panksepp J, Burgdorf J. "Laughing" rats and the evolutionary antecedents of human joy? Physiol Behav 2003;79:533-547.

31. Panksepp JB, Jochman KA, Kim JU, Koy JJ, Wilson ED, Chen Q, et al. Affiliative behavior, ultrasonic communication and social reward are influenced by genetic variation in adolescent mice. PLoS One 2007;2: e351.

32. Bicks LK, Koike H, Akbarian S, Morishita H. Prefrontal cortex and social cognition in mouse and man. Front Psychol 2015;6:1805.

33. Croxson PL, Johansen-Berg H, Behrens TE, Robson MD, Pinsk MA, Gross CG, et al. Quantitative investigation of connections of the prefrontal cortex in the human and macaque using probabilistic diffusion tractography. J Neurosci 2005;25:8854-8866.

34. Ongur D, Price JL. The organization of networks within the orbital and medial prefrontal cortex of rats, monkeys and humans. Cereb Cortex 2000;10:206-219.

35. Jeon D, Kim S, Chetana M, Jo D, Ruley HE, Lin SY, et al. Observational fear learning involves affective pain system and Cav1.2 Ca2+ channels in ACC. Nat Neurosci 2010;13:482-488.

36. Jurado-Parras MT, Gruart A, Delgado-Garcia JM. Observational learning in mice can be prevented by medial prefrontal cortex stimulation and enhanced by nucleus accumbens stimulation. Learn Mem 2012;19: 99-106.

37. Kim BS, Lee J, Bang M, Seo BA, Khalid A, Jung MW, et al. Differential regulation of observational fear and neural oscillations by serotonin and dopamine in the mouse anterior cingulate cortex. Psychopharmacology (Berl) 2014;231:4371-4381.

38. Ben-Ami Bartal I, Decety J, Mason P. Empathy and pro-social behavior in rats. Science 2011;334:1427-1430.

39. Mitchell JP. Inferences about mental states. Philos Trans R Soc Lond B Biol Sci 2009;364:1309-1316.

40. de Quervain DJ, Fischbacher U, Treyer V, Schellhammer M, Schnyder U, Buck A, et al. The neural basis of altruistic punishment. Science 2004; 305:1254-1258.

41. Kim S, Matyas F, Lee S, Acsady L, Shin HS. Lateralization of observational fear learning at the cortical but not thalamic level in mice. Proc Natl Acad Sci U S A 2012;109:15497-15501.

42. Cummins DD. How the social environment shaped the evolution of 
mind. Synthese 2000;122:3-28.

43. Kohls G, Chevallier C, Troiani V, Schultz RT. Social 'wanting' dysfunction in autism: neurobiological underpinnings and treatment implications. J Neurodev Disord 2012;4:10.

44. Preuss TM. Do rats have prefrontal cortex? The rose-woolsey-akert program reconsidered. J Cogn Neurosci 1995;7:1-24.

45. Wang F, Zhu J, Zhu H, Zhang Q, Lin Z, Hu H. Bidirectional control of social hierarchy by synaptic efficacy in medial prefrontal cortex. Science 2011;334:693-697.

46. Olsson A, Nearing KI, Phelps EA. Learning fears by observing others: the neural systems of social fear transmission. Soc Cogn Affect Neurosci 2007;2:3-11.

47. Langford DJ, Crager SE, Shehzad Z, Smith SB, Sotocinal SG, Levenstadt JS, et al. Social modulation of pain as evidence for empathy in mice. Science 2006;312:1967-1970.

48. Wang F, Kessels HW, Hu H. The mouse that roared: neural mechanisms of social hierarchy. Trends Neurosci 2014;37:674-682.

49. Jiang-Xie LF, Liao HM, Chen CH, Chen YT, Ho SY, Lu DH, et al. Autismassociated gene Dlgap2 mutant mice demonstrate exacerbated aggressive behaviors and orbitofrontal cortex deficits. Mol Autism 2014;5:32.

50. Takahashi A, Nagayasu K, Nishitani N, Kaneko S, Koide T. Control of intermale aggression by medial prefrontal cortex activation in the mouse. PLoS One 2014;9:e94657.

51. Knapska E, Mikosz M, Werka T, Maren S. Social modulation of learning in rats. Learn Mem 2010;17:35-42.

52. Wohr M, Scattoni ML. Behavioural methods used in rodent models of autism spectrum disorders: current standards and new developments. Behav Brain Res 2013;251:5-17.

53. Guzman YF, Tronson NC, Guedea A, Huh KH, Gao C, Radulovic J. Social modeling of conditioned fear in mice by non-fearful conspecifics. Behav Brain Res 2009;201:173-178.

54. Bredy TW, Barad M. Social modulation of associative fear learning by pheromone communication. Learn Mem 2009;16:12-18.
55. Abrahams BS, Geschwind DH. Advances in autism genetics: on the threshold of a new neurobiology. Nat Rev Genet 2008;9:341-355.

56. Barnard L, Young AH, Pearson J, Geddes J, O’Brien G. A systematic review of the use of atypical antipsychotics in autism. J Psychopharmacol 2002;16:93-101.

57. Crawley JN. Mouse behavioral assays relevant to the symptoms of autism. Brain Pathol 2007;17:448-459.

58. Lazaro MT, Golshani P. The utility of rodent models of autism spectrum disorders. Curr Opin Neurol 2015;28:103-109.

59. Moy SS, Nadler JJ, Young NB, Perez A, Holloway LP, Barbaro RP, et al. Mouse behavioral tasks relevant to autism: phenotypes of 10 inbred strains. Behav Brain Res 2007;176:4-20.

60. Winslow JT, Hearn EF, Ferguson J, Young LJ, Matzuk MM, Insel TR. Infant vocalization, adult aggression, and fear behavior of an oxytocin null mutant mouse. Horm Behav 2000;37:145-155.

61. Amodio DM, Frith CD. Meeting of minds: the medial frontal cortex and social cognition. Nat Rev Neurosci 2006;7:268-277.

62. Johnson SC, Baxter LC, Wilder LS, Pipe JG, Heiserman JE, Prigatano GP. Neural correlates of self-reflection. Brain 2002;125:1808-1814.

63. Mitchell JP, Macrae CN, Banaji MR. Dissociable medial prefrontal contributions to judgments of similar and dissimilar others. Neuron 2006; 50:655-663.

64. Wise SP. Forward frontal fields: phylogeny and fundamental function. Trends Neurosci 2008;31:599-608.

65. Craig KD. Social communication of pain enhances protective functions: a comment on Deyo, Prkachin and Mercer (2004). Pain 2004;107:5-6.

66. Lamm C, Decety J, Singer T. Meta-analytic evidence for common and distinct neural networks associated with directly experienced pain and empathy for pain. Neuroimage 2011;54:2492-2502.

67. Singer T, Seymour B, O’Doherty J, Kaube H, Dolan RJ, Frith CD. Empathy for pain involves the affective but not sensory components of pain. Science 2004;303:1157-1162. 